\title{
Análisis de supervivencia. Aplicación en una muestra de mujeres con cáncer cervical en México*
}

Lourdes Flores-Luna, M. en C., (1) Salvador Zamora Muñoz, M. en C., (1,2)

Eduardo Salazar-Martínez, D r. en C., (1) Eduardo Lazcano-Ponce, Dr. en C. ${ }^{(1)}$

E 1 análisis de supervivencia es un área estadística en la que la variable respuesta es el tiempo que transcurre entre un evento inicial (que determina la inclusión del individuo en el estudio) y un evento final (genéricamente llamado falla) que ocurre cuando el individuo presenta la característica para terminar el estudio (muerte, alta de la enfermedad, etc.). En este tipo de estudios de cohorte ${ }^{1}$ puede ocurrir que algún individuo lo abandone antes de que le ocurra el evento de interés, registrándose sólo información parcial (censura) sobre la variable de interés (tiempo de falla). El objetivo principal del análisis de supervivencia es incorporar esta información parcial que proporcionan los individuos censurados mediante métodos desarrollados para ese fin. En otras áreas estadísticas esta información parcial es ignorada (datos faltantes); esta manera de proceder es incorrecta, ya que se desconoce el aporte parcial de estos individuos al estudio, lo que es contrario a la filosofía estadística de incorporar toda la información disponible dentro del análisis. Los métodos estadísticos en el análisis de supervivencia son similares a los que se utilizan en otras áreas donde no se presentan datos censurados, por ejemplo: análisis descriptivo, comparación de poblaciones, modelos tipo regresión, etcétera.

Los tipos de censura más comunes en esta área son:

1. Censura tipo I. En muchos estudios el investigador debe determinar un tiempo máximo de obser- vación para que ocurra la falla en los individuos, por lo que aquellos a los cuales aún no les ocurría la falla al concluir el periodo de observación representan una censura.

2. Censura tipo II. En este caso el investigador decide prolongar el periodo de observación hasta que ocurran $k$ fallas de $n$ posibles $(k<n)$, registrando este último valor de falla para el resto de los individuos (censuras) que no observó. Una razón común para determinar el número de fallas a observar es la potencia que se requiere para el estudio. En estos dos casos, la censura está controlada por el investigador.

3. Censura aleatoria. En este tipo de censura el investigador no tiene ningún control sobre la misma. Las censuras pueden ocurrir porque el individuo abandona el estudio, muere por una causa que no es de interés o permanece vivo al término del mismo.

Estos tres tipos de censura son clasificados como censura por la derecha, cuya característica es que el tiempo de censura observado es menor que el tiempo de falla, sólo que este último no es observado.

El objetivo de este capítulo es mostrar el uso de las técnicas del análisis de supervivencia para medir el impacto de los factores que determinan una mayor supervivencia en las mujeres con cáncer cervicouterino (CACU) de un hospital de ginecobstetricia de la ciudad de México. Como ejemplo se utilizó una cohor-

* Este estudio fue realizado con financiamiento del Instituto N acional de Salud Pública (IN SP).

(1) Centro de Investigación en Salud Poblacional, IN SP. Cuernavaca, Morelos, México.

(2) Instituto de Investigación en Matemáticas A plicadas y en Sistemas, Universidad N acional Autónoma de México.

Solicitud de sobretiros: Dr. Eduardo Lazcano Ponce. Centro de Investigación en Salud Poblacional, Instituto N acional de Salud Pública. Avenida Universidad 655, colonia Santa María A huacatitlán, 62508 Cuernavaca, Morelos, México.

Correo electrónico: elazcano@ insp3.insp.mx 
te histórica mediante revisión de expedientes clínicos, durante el periodo de 1984 a 1996 (150 meses), en 378 mujeres en el Hospital de Ginecobstetricia No. 4 Luis Castelazo Ayala, del Instituto Mexicano del Seguro Social, en la ciudad de México, con diagnóstico de CACU invasor confirmado histopatológicamente. Para la obtención de la información se utilizaron entrevistadoras estandarizadas que se mantuvieron ciegas a la hipótesis del estudio. La información se obtuvo mediante un cuestionario diseñado específicamente para el estudio y se tomó como fuente de información los registros clínicos del departamento de ginecología oncológica del hospital. La calidad de la información fue supervisada por dos oncólogos durante el periodo de seguimiento.

Población de estudio. Se seleccionó una unidad de alto nivel de atención que concentra pacientes ginecobstétricas, que cuenta con el servicio de ginecología oncológica, siendo, además, este hospital el que mayor número de casos de CACU notifica en el área metropolitana de la ciudad de México. Las mujeres que han sido canalizadas, diagnosticadas y tratadas en este hospital con CACU invasor confirmadas histopatológicamente fueron elegidas para formar parte del estudio, garantizando así que todas las mujeres son de la misma cohorte y que su entrada al estudio depende del diagnóstico, independientemente de la fecha del mismo.

Definición de las variables de estudio. La variable respuesta fue construida tomando en cuenta el tiempo que transcurre desde el momento del diagnóstico de CACU confirmado histopatológicamente (evento inicial), hasta la ocurrencia de la muerte (evento final). La variable de censura fue definida por las mujeres que no presentaron el evento de interés dentro del periodo de estudio, y como mecanismos de censura fueron establecidos: a) muerte por otra causa diferente al CACU; b) pérdida durante el seguimiento (cambio de domicilio no notificado, negativa a continuar dentro del estudio, falta de seguimiento del tratamiento), y c) todas aquellas mujeres que permanecieron vivas hasta el final del periodo de estudio.

Métodos estadísticos utilizados. Como en muchas áreas estadísticas, los métodos que se utilizan en análisis de supervivencia son tanto paramétricos como no paramétricos; entendiéndose por estos últimos, aquellos métodos que no suponen ninguna distribución sobre los datos observados. Dado el objetivo que se perseguía en esta investigación se utilizaron únicamente métodos no paramétricos, a excepción del modelo de riesgos proporcionales (MRP) que es semiparamétrico. A continuación se describen brevemente:
Tabla de vida. Esta tabla es un resumen (parecido a la tabla de frecuencias usual) de la información recabada. Contiene algunas estadísticas que permiten tener una visión, en cada intervalo de tiempo, del comportamiento de la mortalidad, supervivencia y tasa de riesgo, entre otras características de las pacientes en la muestra. $^{2}$

Un indicador importante del impacto que puede tener el CACU sobre las pacientes es la experiencia de supervivencia de la población; entendiéndose a ésta como la probabilidad de que una paciente sobreviva a un tiempo fijo, $t$. El uso del estimador Kaplan-Meier (K-M), permite estimar los valores de la supervivencia en cualquier momento y proporciona, además, una manera gráfica de presentarla. ${ }^{3}$ En caso de que se deseen comparar varias poblaciones, una manera de explorar si existe diferencia entre ellas, al menos de manera empírica, es graficar simultáneamente los estimadores de K-M.

Para evaluar el efecto que cada uno de los factores pronósticos tienen sobre la supervivencia de la población es común realizar comparaciones univariadas entre las poblaciones que determinan las categorías de estos factores, por lo general, por medio de pruebas como las de log-rank y Wilcoxon. ${ }^{4}$

Modelo de riesgos proporcionales. Una manera usual de determinar el efecto conjunto de los factores pronósticos que resultan significativos de forma individual es mediante el modelo de regresión de $\mathrm{Cox}^{5} \mathrm{o}$ modelo de riesgos proporcionales. Este modelo especifica cómo cambia la función de riesgo básica (individuos con nivel de covariables cero) respecto de aquellos con covariables distintas de cero. Este cambio lo especifica el parámetro asociado a cada factor introducido al modelo y se interpreta como el cambio esperado en el cociente de riesgos entre un individuo en la población básica y uno fuera de ella.

Los supuestos del modelo de riesgos proporcionales de Cox son los siguientes:

1. La razón de riesgos, para cualquier variable $X$, es constante a través del tiempo.

2. En términos de las probabilidades de supervivencia, la curva de supervivencia de un grupo debe estar siempre por encima de la curva de supervivencia del otro grupo; éstas no se pueden cruzar.

3. Si el supuesto es violado, entonces se debe utilizar el modelo de Cox estratificado.

4. El supuesto de proporcionalidad se evalúa mediante: a) las líneas del gráfico de las curvas de 
supervivencia (éstas no deben cruzarse), y b) las líneas del gráfico loglog: $\operatorname{Ln}[-\operatorname{Ln}(\mathrm{S})]$ vs. tiempo para todos los grupos (las líneas deben ser aproximadamente paralelas).

Después de ajustar el MRP es importante verificar si éste se ajusta adecuadamente a los datos, por lo que se utilizan distintas clases de residuos, que sirven para verificar diversas características propias del MRP. $^{6-8}$

Como última fase del análisis, es muy importante evaluar el impacto de cada observación en el ajuste del MRP. Las observaciones pueden producir un fuerte impacto en todo el vector de parámetros (una observación que tiene un impacto de esta naturaleza, implica que el modelo cambia radicalmente con y sin la observación) o en particular, en cada una de las covariables que componen el modelo. ${ }^{9}$ En cualquiera de estos dos casos es importante, después de localizar estas observaciones, tratar de determinar a qué se debe lo desmedido de su impacto en el MRP. Cabe destacar que cada uno de los métodos descritos incorpora las censuras en su construcción.

Para el manejo y análisis de la información se utilizó el paquete estadístico Stata 6.0.

\section{Resultados}

El cuadro I muestra la tabla de vida. En ella se observa que la mayoría de las muertes por CACU ocurren en los primeros tres años de observación (92 de 107), lo que da como resultado que la probabilidad de sobrevivir a esos tres años se reduzca en $28 \%$, que es muy alta comparada con la reducción total que es de $56.54 \%$ (en términos relativos es aproximadamente
$50 \%$ ). Por supuesto, lo anterior se ve reforzado con el hecho de que en este periodo se tienen las tasas de riesgo, en general, más elevadas (se puede consultar el apéndice para observar cómo cambia la supervivencia de acuerdo con el estadio clínico de la enfermedad).

La figura1 muestra la gráfica del estimador K-M. Aquí podemos observar de manera más precisa el decrecimiento de la supervivencia en los tres primeros años (1 000 días aproximadamente). Los años siguientes muestran una caída en la supervivencia mucho más lenta, además de periodos en los que ésta se mantiene constante.

El cuadro II resume los datos de supervivencia estimados. Encontramos que las mujeres que se habían realizado alguna vez la prueba de Papanicolaou tienen una supervivencia de $75.65 \%$, que es estadísticamente superior a la de las que nunca se lo habían hecho $(46 \%)$ (las pruebas de log-rank mostraron diferencias en las curvas, $p<0.001)$. Para la etapa I se tiene una supervivencia de $82 \%$ y desciende al aumentar la etapa clínica, encontrando que para las etapas tardías la supervivencia fue de $47 \%$ en la etapa III, y de $21 \%$ en la etapa IV $(p<0.001)$. La presencia de síntomas se clasificó de la siguiente manera:

1. Generales, esta categoría está formada por las mujeres que no presentaron ningún síntoma o únicamente tuvieron descarga vaginal.

2. Específicos, abarcan: a) hemorragia genital anormal (HGA); b) HGA poscoito, y c) dolor.

3. Graves, se definieron así cuando existe tumor pélvico o pérdida de peso.

Al evaluar la presencia de síntomas, encontramos que cuando hay síntomas graves se muestra la menor

\section{Tabla de vida de las mujeres con cáncer Cervicouterino. Hospital de Ginecobstetricia No. 4, MÉxıCo, 1984-1996}

\begin{tabular}{|c|c|c|c|c|c|c|c|}
\hline $\begin{array}{l}\text { Intervalo } \\
\text { (años) }\end{array}$ & $\begin{array}{l}\text { Número de mujeres } \\
\text { en el intervalo }\end{array}$ & $\begin{array}{l}\text { Número de } \\
\text { censuras }\end{array}$ & $\begin{array}{l}\text { Número de mujeres } \\
\text { expuestas al riesgo }\end{array}$ & Fallas & $\begin{array}{l}\text { Proporción } \\
\text { de fallas }\end{array}$ & $\begin{array}{l}\text { Supervivencia } \\
\text { acumulada }\end{array}$ & $\begin{array}{l}\text { Función } \\
\text { de riesgo }\end{array}$ \\
\hline $0-1$ & 378 & 27 & 364.5 & 59 & .1619 & .8381 & .0005 \\
\hline $1-2$ & 292 & 62 & 261.0 & 19 & .0728 & .7771 & .0002 \\
\hline $2-3$ & 211 & 41 & 190.5 & 14 & .0735 & .7200 & .0002 \\
\hline $3-4$ & 156 & 49 & 131.5 & 3 & .0228 & .7036 & .0001 \\
\hline $4-5$ & 104 & 33 & 87.5 & 5 & .0571 & .6634 & .0002 \\
\hline $5-6$ & 66 & 25 & 53.5 & 2 & .0374 & .6386 & .0001 \\
\hline $6-7$ & 39 & 15 & 31.5 & 0 & .0000 & .6386 & .0000 \\
\hline $7-8$ & 24 & 7 & 20.5 & 1 & .0488 & .6074 & .0001 \\
\hline $8-9$ & 16 & 7 & 12.5 & 1 & .0800 & .5588 & .0002 \\
\hline $9-10$ & 8 & 3 & 6.5 & 0 & .0000 & .5588 & .0000 \\
\hline $10-11$ & 5 & 1 & 4.5 & 1 & .2222 & .4346 & .0007 \\
\hline 11-12 & 3 & 1 & 2.5 & 0 & .0000 & .4346 & .0000 \\
\hline $12-13$ & 2 & 2 & 1.0 & 0 & .0000 & .4346 & .0000 \\
\hline
\end{tabular}




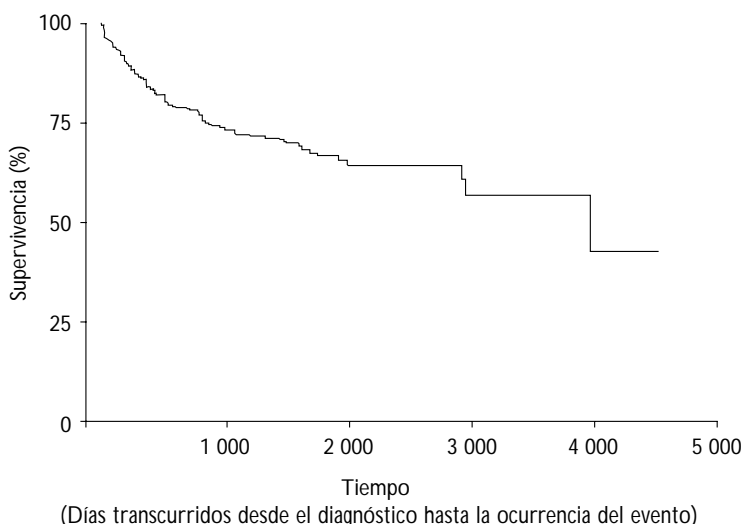

Figura 1. Estimador de Kaplan-Meier, curva de SUPERVIVENCIA GLOBAL DE MUJERES CON CÁNCER Cervicouterino. Hospital de Ginecobstetricia No.4, Méxıco, 1984-1996

supervivencia $(50.26 \%)$, aumentando ésta cuando la paciente presentaba síntomas específicos $(66.80 \%)$ y hasta $85.45 \%$ cuando sólo existían síntomas generales con diferencia entre ellos $(p<0.001)$. El tipo adenoescamoso presentó la menor supervivencia (53.04\%). Al evaluar el tamaño tumoral encontramos que tumores mayores de seis centímetros tienen una tasa de supervivencia más baja (39.74\%) que los tumores más pequeños (79.62\%; $p<0.001)$. Cuando la invasión es linfovascular la supervivencia es de $27.71 \%(p<0.001)$.

El cuadro III muestra los factores pronósticos que resultaron significativos en el MRP, encontrando que las etapas tardías del tumor, tales como la etapa III y la etapa IV aumentan el riesgo de morir por este padecimiento aproximadamente dos y tres veces, respectivamente. En cuanto a síntomas, el riesgo más alto lo tienen la presencia de síntomas graves, como pérdida de peso, con un aumento tres veces mayor respecto a los síntomas generales. El tipo histológico adenoescamoso, incrementa el riesgo de morir más del doble. Un factor de protección importante es la realización de una prueba de Papanicolaou, ya que disminuye a la mitad el riesgo de morir por esta enfermedad.

Otros factores estudiados que no contribuyeron con información significativa fueron: la escolaridad, los antecedentes familiares de cáncer, el número de cigarrillos al día, la edad de la menarca, la edad de inicio de vida sexual, el número de partos, el número de parejas sexuales, el grado de diferenciación, patrón clínico, tamaño del tumor, diseminación y tratamiento (datos no mostrados en tablas).

\section{Cuadro II \\ SUPERVIVENCIA DE LAS MUJERES CON CÁnCER CERVICOUTERINO. Hospital de GineCobstetricia No. 4, Méxıco, 1984-1996}

Variable

Supervivencia Valor estimada a los de $p^{\ddagger}$ cinco años (\%)*

Realización de la prueba de Papanicolaou

\begin{tabular}{lll} 
No & 46.00 & \\
\hline Sí & 75.65 & $<0.001$
\end{tabular}

Presentación de síntomas

\begin{tabular}{lll} 
Síntomas generales & 85.45 & \\
\hline Síntomas específicos & 66.80 & \\
\hline Síntomas graves & 50.26 & $<0.001$
\end{tabular}

Etapa clínica

\begin{tabular}{lll} 
I & 82.00 & \\
\hline II & 72.72 & \\
\hline III & 47.00 & \\
\hline IV & 21.00 & $<0.001$
\end{tabular}

Tipo histológico

\begin{tabular}{lll} 
Epidermoide & 66.60 & \\
\hline Adenocarcinoma & 74.73 & \\
\hline Adenoescamoso & 53.04 & 0.151
\end{tabular}

Tamaño del tumor $(\mathrm{cm})$

\begin{tabular}{lll}
-2.00 & 79.62 & \\
\hline $2.01-4.00$ & 70.40 & \\
\hline $4.01-6.00$ & 70.78 & \\
\hline$\cdot 6.01$ & 39.74 & $<0.001$
\end{tabular}

Diseminación

Sin invasión a tejidos adyacentes o distantes $\quad 77.20$

\begin{tabular}{lll} 
Invasión de tejidos regionales & 64.93 & \\
\hline Invasión linfovascular & 27.71 & $<0.001$
\end{tabular}

* Método Kaplan-Meier

₹ Prueba de log-rank

Validación del modelo de riesgos proporcionales. La figura 2 presenta los residuos de Cox-Snell contra su función de riesgo acumulada. En ella se puede observar aproximadamente una línea recta con pendiente uno que pasa por el origen, que indica un buen ajuste del modelo.

En el cuadro IV se presentan tanto la evaluación global del supuesto de riesgos proporcionales como la evaluación del mismo por cada covariable dentro del 


\section{Cuadro III}

FACTORES PRON ÓSTICOS EN LAS MUJERES CON CÁNCER Cervicouterino. Hospital de Ginecobstetricia No.4, MÉxıco, 1984-1996

Variable

Modelo de riesgos proporcionales

Realización de la prueba de Papanicolaou

\begin{tabular}{ccc} 
No & $1.00^{\ddagger}$ & \\
\hline Sí & 0.46 & $0.30-0.70$
\end{tabular}

Etapa clínica

\begin{tabular}{ccc} 
I & $1.00^{\ddagger}$ & \\
\hline II & 1.02 & $0.57-1.85$ \\
\hline III & 1.93 & $1.08-3.44$ \\
\hline IV & 5.99 & $2.83-12.67$
\end{tabular}

Presentación de síntomas

\begin{tabular}{lll} 
Síntomas generales & $1.00^{\ddagger}$ & \\
\hline Síntomas específicos & 2.50 & $0.98-6.36$ \\
\hline Síntomas graves & 3.26 & $1.21-8.72$
\end{tabular}

Tipo histológico

\begin{tabular}{lll} 
Adenocarcinoma & $1.00^{\ddagger}$ & \\
\hline Epidermoide & 1.38 & $0.75-2.52$ \\
\hline Adenoescamoso & 2.24 & $1.05-4.78$
\end{tabular}

* Razón de riesgos del MRP, ajustada por las variables incluidas en el modelo

₹ Categoría de referencia

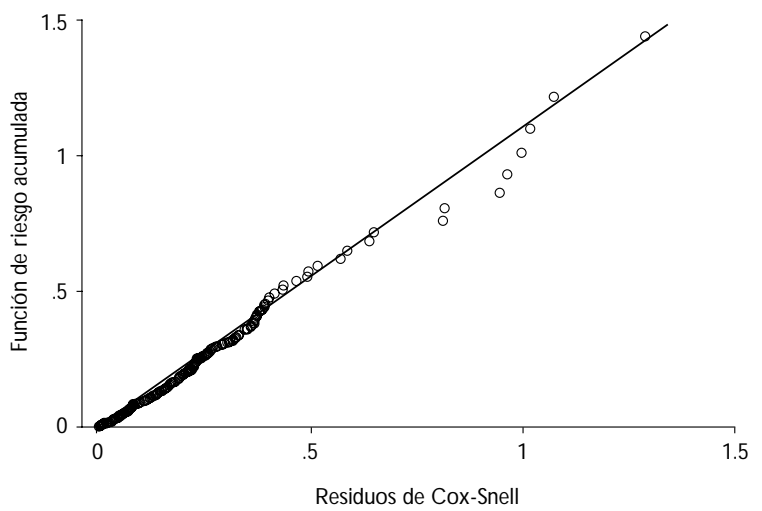

Figura 2. Evaluación del ajuste del modelo de RIESGOS PROPORCIONALES, EN UNA MUESTRA DE MUJERES CON CÁNCER CERVICOUTERINO. HOSPITAL DE Ginecobstetricia No. 4, México, 1984-1996 modelo (utilizando los residuos de Schoenfeld). La hipótesis nula especifica que los riesgos son proporcionales (gráficamente se ven como curvas paralelas desplazadas una respecto de las otras, figura 3), contra la alternativa de que al menos uno no lo es. Los resultados muestran que en forma global se cumple este supuesto ( $p=0.1595$ ) y las variables que, en forma individual, mejor sustentan este supuesto son tipo histológico y etapa, respectivamente.

La gráfica de los residuos de devianza (figura 4) muestra que se cumplen los supuestos de homogeneidad de varianza y no correlación de los residuos. Los residuos de martingalas (figura 5) evidencian que es correcto el supuesto de incluir de forma lineal las variables en el modelo. Aunque en este caso no resultan muy ilustrativas porque las variables utilizadas son categóricas (sólo se muestran para etapa). Ambas gráficas permiten detectar algunas observaciones con residuos grandes, mismos que serán analizados con detalle posteriormente.

Diagnóstico. En la evaluación global del impacto de las observaciones sobre el MRP, el estadístico utilizado muestra que la observación 366 tiene un impacto muy grande, 0.7680914 (esta estadística está estandarizada, de tal manera que la suma de los cuadrados de sus valores sea uno y la aportación de esta observación es de 0.5899$)$. Al quitar la observación y correr el modelo sin la misma, sólo hubo un cambio notorio en el coeficiente asociado a etapa IV (5.996535 a 8.109502).

\section{Cuadro IV}

Evaluación gLobal y POR COVARIABLE DEL SUPUESTO DE RIESGOS PROPORCIONALES, EN UNA MUESTRA DE MUJERES CON CÁNCER CERVICOUTERINO. Hospital de Ginecobstetricia No. 4, MÉxıco, 1984-1996

\begin{tabular}{ll} 
Variable & Valor de $\mathrm{p}^{*}$ \\
Papanicolaou & 0.0578 \\
\hline Síntomas específicos & 0.0278 \\
\hline Síntomas graves & 0.0595 \\
\hline Epidermoide & 0.5989 \\
\hline Adenoescamoso & 0.9071 \\
\hline Etapa II & 0.2983 \\
\hline Etapa III & 0.2258 \\
\hline Etapa IV & 0.8081 \\
Evaluación global & \\
$*$ Prueba de $\chi^{2}$ & 0.1595 \\
\hline
\end{tabular}




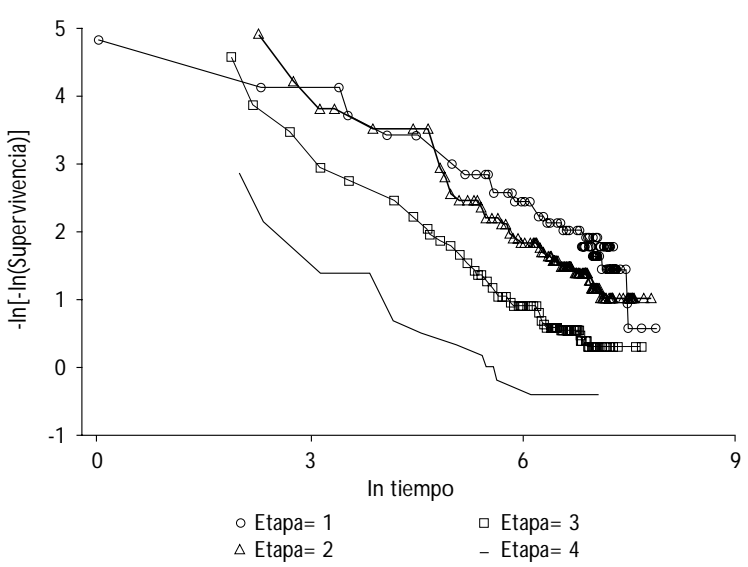

Figura 3. Riesgos PRoporcionales POR ETA PA, EN UNA MUESTRA DE MUJERES CON CÁNCER CERVICOUTERINO. Hospital de Ginecobstetricia No. 4, México, 19841996

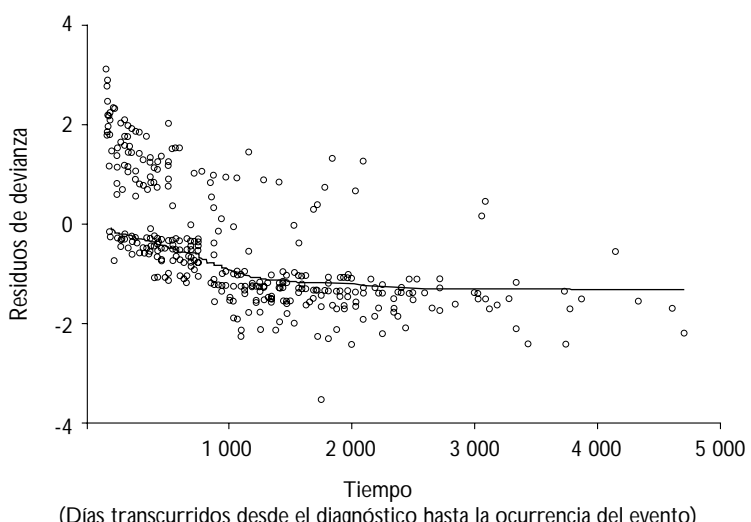

Figura 4. Evaluación de los supuestos del modelo DE RIESGOS PROPORCIONALES, EN UNA MUESTRA DE MUJERES CON CÁNCER CERVICOUTERINO. HOSPITAL DE Ginecobstetricia No. 4, México, 1984-1996

Si se realiza un análisis detallado de los registros para esta observación se encuentra que la característica que puede hacer atípica a esta paciente es el hecho de que pertenece a la etapa IV (etapa tardía) y su registro de supervivencia es grande comparado con el promedio del resto de observaciones en esa categoría (347 días vs. 1650 días). No obstante lo anterior, se decidió conservar el modelo original, ya que, pese a lo poco común de esta observación, es posible que ocu-

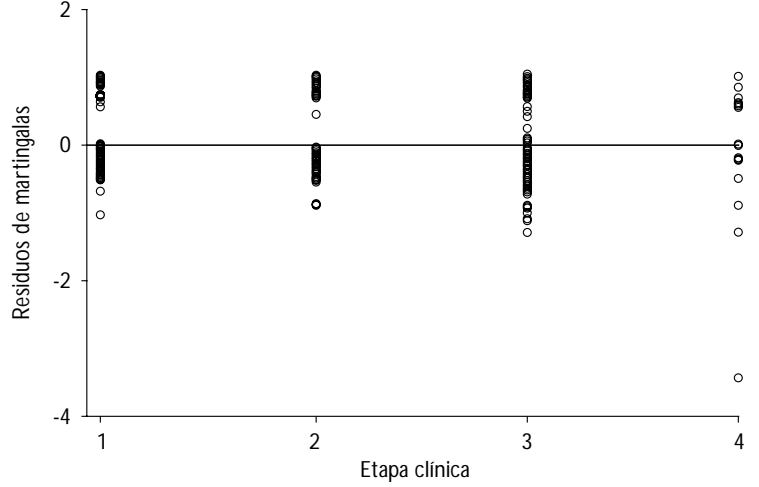

Figura 5. Evaluación de la linealidad del factor PRONÓSTICO, EN UNA MUESTRA DE MUJERES CON CÁNCER Cervicouterino. Hospital de Ginecobstetricia N o. 4, Méxıco, 1984-1996

rra y, por lo tanto, no existe ningún argumento para eliminarla.

$\mathrm{Al}$ evaluar el impacto de las observaciones sobre las covariables en el modelo se encontró que el más fuerte lo ejerce la observación 366 sobre la variable etapa IV, confirmando así el resultado encontrado en la evaluación global.

\section{Conclusiones}

El uso de los métodos del análisis de supervivencia, a lo largo de este artículo, ha puesto de manifiesto lo importante que resulta utilizar los procesos de análisis de información más adecuados de acuerdo con las características de los datos. Como se comentó en la introducción, estos métodos incorporan las observaciones censuradas en su construcción, lo que permite tener una descripción más adecuada de las mujeres mexicanas con CACU, así como la evaluación de los factores pronósticos que determinan su supervivencia. En este último punto, es fundamental que los métodos utilizados incorporen las censuras, ya que, de lo contrario, se corre el riesgo de introducir un sesgo en la estimación de estos efectos en la población.

Además de describir a la población, hemos podido evaluar el efecto que de manera individual tienen algunos de estos factores pronósticos sobre la supervivencia, mediante pruebas como log-rank y Wilcoxon. Sin embargo, estas pruebas no permiten hacer una evaluación conjunta de todos los factores que fueron significativos para esta supervivencia, por lo que se utilizó el MRP que permite evaluar el impacto de un factor, 
considerando la intervención de otros de forma simultánea. Esta evaluación conjunta es muy importante, ya que, como se observó en el análisis, no necesariamente todos los factores que son significativos de forma individual lo son cuando se introducen conjuntamente en el MRP. Además este modelo permite evaluar el impacto que tiene un factor en la supervivencia, comparando las poblaciones por medio del cociente de los riesgos respectivos.

En nuestro caso, el MRP ha permitido determinar que la supervivencia de las mujeres mexicanas con CACU está determinada principalmente por cuatro factores: a) la realización de la prueba de Papanicolaou; b) la etapa clínica; c) los síntomas, y d) el tipo histológico. Estos factores también se han detectado en otros estudios en el mundo. ${ }^{10-21}$

Como quedó de manifiesto anteriormente, es muy importante evaluar mediante un procedimiento estadístico, que el MRP se ajusta adecuadamente a nuestros datos, de lo contrario, las asociaciones encontradas entre los factores pronósticos y las inferencias a la población serían cuestionables. En nuestro caso, pudimos dar evidencia de que el modelo se ajusta bien a los datos observados y los supuestos se cumplen razonablemente bien.

De suma importancia para completar el análisis del ajuste del MRP, es la evaluación del impacto que cada observación tiene sobre él. En nuestro estudio, el impacto de la observación 366 sólo se manifestó en la estimación de un parámetro, pero pueden darse casos en los que una observación de esta naturaleza modifique de manera sustancial todo el modelo, por lo que resulta muy importante cubrir esta etapa del análisis.

Finalmente, el uso de herramientas propias del análisis de supervivencia nos ha permitido conocer los factores pronósticos que inciden de manera importante en la expectativa de supervivencia de las mujeres mexicanas con CACU, y los resultados encontrados coinciden, en general, con los que se han notificado en estudios previos, tanto con el tipo de factores como con el impacto de los mismos.

\section{A gradecimientos}

Se agradece la participación del personal del servicio de ginecología oncológica del Hospital de Ginecobstetricia No. 4 Luis Castelazo Ayala, del Instituto Mexicano del Seguro Social, en la ciudad de México. Especialmente a los doctores Guillermo González Lira y Pedro Escudero de los Ríos.

\section{Referencias}

1. Mantel N, Haenszel W. Statistical aspects of the analysis of data from retrospective studies of disease. . N atl Cancer Inst 1959;22:719-748.

2. Collet $D$. Modelling survival data in medical research. Londres: $C$ hapman \& Hall, 1996.

3. Kaplan EL, Meier P. N onparametric estimation from incomplete observations. J Am Stat Assoc 1958:53:457-481.

4. Mantel $N$. Evaluation of survival data and two new rank order statistics arising in its consideration. Cancer Chemother Rep 1966;50:163-170.

5. Cox DR. Regression models and life tables. J R Stat Soc 1972;74(B): 187-220.

6. Cox DR, Snell EJ. A general definition of residuals. J $R$ Stat Soc 1968:30(A):248-275.

7. Schoenfeld DA. Chi-squared goodness of fit test for the proportional hazards regression model. Biometrika 1980;67:145-153.

8. Schoenfeld DA. Partial residuals for the proportional hazards regression model. Biometrika 1982;69:239-241.

9. Weissfeld LA, Schneider H. Influence diagnostics for the proportional hazards model. Stat Prob Lett 1990;10:411-417.

10. Kosary CL. FIGO stage, histology, histologic grade, age and race as prognostic factors in determining survival for cancers of the female gynecological system:An analysis of 1973-1987 SEER cases of cancers of the endometrium, cervix, ovary, vulva and vagina. Semin Surg 0 ncol 1994;10: 31-46.

11. Yoshioka T, Inoue Y, H oshiaia H, N oda K. Prognostic factors in uterine cervical and corpus cancer. Gan to Kagaku Ryoho 1995;22:1417-1422.

12.W erner-W asik M, Schmid CH, Bornstein L, Ball HG, Smith D M, MadocJones $\mathrm{H}$. Prognostic factors for local and distant recurrence in stage I and II cervical carcinoma. Int J Radiat O ncol Biol Phys 1995;32:1309-1317.

13. Pedersen $D$, So gaard $H, O$ vergaard J, Bentzen SM. Prognostic value of pretreatment factors in patients with locally advanced carcinoma of the uterine cervix treated by radiotherapy alone.Acta 0 ncol 1995;34: 787-795.

14. A charki A, Sahraoui S, Benider A, Tawfiq N, Jouhadi H, Boutas N et al. $C$ ancer of the uterine cervix in young women. $A$ retrospective study of 337 cases. Bull Cancer 1997;84:373-378.

15. Perez CA, G rigsby PW, Camel HM, Galakatos AE, Mutch D, Lockett $M A$. Irradiation alone or combined with surgery in stage IB, IIA and IIB carcinoma of uterine cervix:Update of a non-randomized comparison. Int J Radiat O ncol Biol Phys 1995;31:703-716.

16. Jones W, Shingleton $H$, Russell A, Chmiel J, Fremgen A, Clive R et al. Patterns of care for invasive cervical cancer. Cancer 1995;76:1934-1947. 17. Shingleton H, Bell M, Fremgen A, Chmiel J, Rusell A, Jones W et al. Is there really a diference in survival of women with squamous cell carcinoma, adenocarcinoma and adenosquamous cell carcinoma of the cervix? Cancer 1995;76:1948-1955.

18. Hopkins M, Morley G. Prognostic factors in advanced stage squamous cell cancer of the cervix. Cancer 1993;72:2389-2393.

19. Bernd-U we S, Mehrdad N , Lampe B, LuY, Hilsenbeck S, Koecjli 0 et al. Prognostic factors of early stage cervical cancer treated by radical hysterectomy. Cancer 1995;76:1978-1986.

20. Perez CA, G rigsby PW, N ene M, C amel HM, Galakatos A, Kao M et al. Efect of tumor size on the prognosis of carcinoma of the uterine cervix treated with irradiation alone. Cancer 1992;69:2796-2806.

21. Dattoli M, Herbert F, Beller U, Lerch I, D emopoulos R, Beckman E et al. A nalysis of multiple prognostic factors in patients with stage IB cancer:Age as mayor determinant. Int J Radiat O ncol Biol Phys 1989;17(1): 41-47. 


\section{A péndice}

El objetivo de este apartado es presentar por medio de tablas de vida las etapas del tumor, así como ilustrar el cálculo de algunos de sus elementos.

\section{TABLA DE VIDA DE LAS MUJERES CON CÁNCER CERVICOUTERINO}

\begin{tabular}{|c|c|c|}
\hline $\begin{array}{l}\text { Intervalo } \\
\text { (años) }\end{array}$ & $\begin{array}{l}\text { Número de mujeres } \\
\text { en el intervalo }\end{array}$ & $\begin{array}{l}\text { Número de } \\
\text { censuras }\end{array}$ \\
\hline
\end{tabular}

\begin{tabular}{cccccccc} 
Etapa I & & & & & & & \\
$0-1$ & 126 & 14 & 119.0 & .0840 & .000 & .0002 \\
\hline $1-2$ & 102 & 26 & 89.0 & 4 & .0449 & .8748 & .0001 \\
\hline $2-3$ & 72 & 15 & 64.5 & 1 & .0155 & .8612 & .0000 \\
\hline $3-4$ & 56 & 12 & 50.0 & 1 & .0200 & .8440 & .0001 \\
\hline $4-5$ & 43 & 12 & 37.0 & 1 & .0270 & .8212 & .0001 \\
\hline $5-6$ & 30 & 12 & 24.0 & 1 & .0417 & .7870 & .0001
\end{tabular}

Etapa II

\begin{tabular}{lccccccc}
$0-1$ & 137 & 10 & 132.0 & 14 & .1061 & .8939 & .0003 \\
\hline $1-2$ & 113 & 22 & 102.0 & 5 & .0490 & .8501 & .0001 \\
\hline $2-3$ & 86 & 17 & 77.5 & 5 & .0645 & .7953 & .0002 \\
\hline $3-4$ & 64 & 23 & 52.5 & 1 & .0190 & .7801 & .0001 \\
\hline $4-5$ & 40 & 15 & 32.5 & 2 & .0615 & .7321 & .0002 \\
\hline $5-6$ & 23 & 7 & 19.5 & 1 & .0513 & .6946 & .0001
\end{tabular}

Etapa III

\begin{tabular}{lrrrrrrr}
$0-1$ & 97 & 2 & 96.0 & 24 & .2500 & .7500 & .0008 \\
\hline $1-2$ & 71 & 13 & 64.5 & 8 & .1240 & .6570 & .0004 \\
\hline $2-3$ & 5 & 46.0 & 8 & .1739 & .5427 & .0005 \\
\hline $3-4$ & 34 & 14 & 27.0 & 1 & .0370 & .5226 & .0001 \\
\hline $4-5$ & 19 & 5 & 16.5 & 2 & .1212 & .4593 & .0004 \\
\hline $5-6$ & 12 & 5 & 9.5 & 0 & .0000 & .4593 & .0000
\end{tabular}

Etapa IV

\begin{tabular}{llllllll}
$0-1$ & 18 & 1 & 17.5 & 11 & .6286 & .3714 & .0025 \\
\hline $1-2$ & 6 & 1 & 5.5 & 2 & .3636 & .2364 & .0012 \\
\hline $2-3$ & 3 & 1 & 2.5 & 0 & .0000 & .2364 & .0000 \\
\hline $3-4$ & 2 & 0 & 2.0 & 0 & .0000 & .2364 & .0000 \\
\hline $4-5$ & 2 & 1 & 1.5 & 0 & .0000 & .2364 & .0000 \\
\hline $5-6$ & 1 & 1 & 0.5 & 0 & .0000 & .2364 & .0000
\end{tabular}


Antes de iniciar los cálculos correspondientes incorporaremos alguna notación. Para cada tabla definiremos como:

$d_{j}$ : número de fallas (muertes) en el intervalo $j$

$c_{i}:$ número de censuras en el intervalo $j$

$n_{j}^{\prime}=d_{j}-\frac{c_{j}}{2}$ : número de individuos en riesgo en el intervalo $\mathrm{j}$ (aquí se supone que los individuos que se censuran en este intervalo estuvieron en riesgo la mitad del mismo)

$\hat{\mathrm{S}}_{\mathrm{j}}=\prod_{i=1}^{j} \stackrel{d_{j}}{ }-\frac{}{n_{j}}$ supervivencia en el intervalo j,i.e., la probabilidad de sobrevivir al intervalo j, dado que se llegó vivo al inicio del mismo

$\hat{\mathrm{h}}_{\mathrm{j}}=\frac{d_{j}}{d_{j}}:$ función de riesgo estimada en el intervalo $\mathrm{j}$

$\left(n_{j}^{\prime}-\frac{-}{2}\right)^{*} \tau$

$\tau: \quad$ Tamaño del intervalo

Ahora, ilustraremos algunos cálculos de estas dos últimas cantidades para cada etapa.

\section{Etapa I}

$\hat{S}=\left(1-\frac{10}{119}\right)=0.9160$

$\hat{S}_{2}=\left(1-\frac{10}{119}\right)^{*}\left(1-\frac{4}{89}\right)=0.9160^{*} 0.9551=0.8748$

$\hat{\mathrm{S}}_{3}=\left(1-\frac{10}{119}\right)^{*}\left(1-\frac{4}{89}\right)^{*}\left(1-\frac{1}{64.5}\right)=0.9160^{*} 0.9551^{*} 0.9845=0.8612$

Que denotan las supervivencias al primero, segundo y tercer año, respectivamente, de las mujeres con CACU en etapa I. De una forma totalmente análoga se calculan el resto de los intervalos hasta el final.

$$
\begin{aligned}
& \hat{\mathrm{h}}_{1}=\frac{10}{\left(119-\frac{10}{2}\right) * 365}=0.0002 \\
& \hat{\mathrm{h}}_{2}=\frac{4}{\left(89-\frac{4}{2}\right) * 365}=0.0001
\end{aligned}
$$

$$
\hat{\mathrm{h}}_{3}=\frac{1}{\left(64.5-\frac{1}{2}\right) * 365}=0.00004
$$

Estas cantidades estiman la función de riesgo en el primero, segundo y tercer año, respectivamente, para las mujeres en esta etapa del tumor, suponiendo que ésta se mantiene constante (el valor estimado) a lo largo de cada año. Los intervalos faltantes se calculan de manera similar.

\section{Etapa II}

$\hat{\mathrm{S}}_{1}=\left(1-\frac{14}{132}\right)=0.8939$

$\hat{S}_{2}=\left(1-\frac{14}{132}\right)^{*}\left(1-\frac{5}{102}\right)=0.8939 * 0.9510=0.8501$

$\hat{\mathrm{S}}_{3}=\left(1-\frac{14}{132}\right)^{*}\left(1-\frac{5}{102}\right)^{*}\left(1-\frac{5}{77.5}\right)=0.8939^{*} 0.9510^{*} 0.9355=0.7953$

$\hat{\mathrm{h}}_{1}=\frac{14}{\left(132-\frac{14}{2}\right) * 365}=0.0003$

$\hat{\mathrm{h}}_{2}=\frac{5}{\left(102-\frac{5}{2}\right)^{*} 365}=0.0001$

$\hat{\mathrm{h}}_{3}=\frac{5}{\left(77.5-\frac{5}{2}\right) * 365}=0.00002$

\section{Etapa III}

$\hat{S}_{1}=\left(1-\frac{24}{96}\right)=0.7500$

$\hat{S}_{2}=\left(1-\frac{24}{96}\right) *\left(1-\frac{8}{64.5}\right)=0.7500 * 0.8760=0.6570$

$\hat{\mathrm{S}}_{3}=\left(1-\frac{24}{96}\right)^{*}\left(1-\frac{8}{64.5}\right)^{*}\left(1-\frac{8}{46}\right)=0.8939^{*} 0.9510^{*} 0.8261=0.5427$

$\hat{\mathrm{h}}_{1}=\frac{24}{\left(96-\frac{24}{2}\right) * 365}=0.0008$ 


$$
\begin{aligned}
& \hat{\mathrm{h}}_{2}=\frac{8}{\left(64.5-\frac{8}{2}\right)^{*} 365}=0.0004 \\
& \hat{\mathrm{h}}_{3}=\frac{8}{\left(46-\frac{8}{2}\right) * 365}=0.00005
\end{aligned}
$$

\section{Etapa IV}

$$
\begin{aligned}
& \hat{\mathrm{S}}_{1}=\left(1-\frac{11}{17.5}\right)=0.3714 \\
& \hat{\mathrm{S}}_{2}=\left(1-\frac{11}{17.5}\right)^{*}\left(1-\frac{2}{5.5}\right)=0.3714^{*} 0.6364=0.2364 \\
& \hat{\mathrm{S}}_{3}=\left(1-\frac{11}{17.5}\right)^{*}\left(1-\frac{2}{5.5}\right) *\left(1-\frac{5}{2.5}\right)=0.3714^{*} 0.6364^{*} 1=0.2364 \\
& \left.\hat{\mathrm{h}}_{1}=\frac{11}{\left(17.5-\frac{11}{2}\right.}\right)^{* 365}=0.0025
\end{aligned}
$$

$$
\begin{aligned}
& \hat{\mathrm{h}}_{2}=\frac{2}{\left(5.5-\frac{2}{2}\right)^{* 365}}=0.0012 \\
& \hat{\mathrm{h}}_{3}=\frac{0}{\left(2.5-\frac{0}{2}\right)^{* 365}}=0.0000
\end{aligned}
$$

Algunos comentarios importantes:

La supervivencia estimada a los cinco años que se presenta en el cuerpo del artículo no se calculó mediante la tabla de vida sino del K-M que es más preciso.

El cálculo de las tablas de vida se realizó en SPSS 8.0 .

Los elementos que se muestran en las tablas no son todos los que se obtienen con este proceso. Se presentaron sólo los que se consideraron importantes en el análisis. No se presenta ningún cálculo de los errores estándar de los valores estimados de la supervivencia ni de la función de riesgo. 